\title{
Enhancement of Josephson phase diffusion by microwaves
}

\author{
Y. Koval, M. V. Fistul* and A. V. Ustinov \\ Physikalisches Institut III, Universität Erlangen-Nürnberg, D-91058, Erlangen, Germany
}

(Dated: November 13, 2018)

\begin{abstract}
We report an experimental and theoretical study of the phase diffusion in small Josephson junctions under microwave irradiation. A peculiar enhancement of the phase diffusion by microwaves is observed. The enhancement manifests itself by a pronounced current peak in the current-voltage characteristics. The voltage position $V_{\text {top }}$ of the peak increases with the power $P$ of microwave radiation as $V_{\text {top }} \propto \sqrt{P}$, while its current amplitude weakly decreases with $P$. As the microwave frequency increases, the peak feature evolves into Shapiro steps with finite slope. Our theoretical analysis taking into account the enhancement of incoherent superconducting current by multi-photon absorption is in good agreement with experimental data.

PACS numbers: $74.50 .+\mathrm{r}, 74.40 .+\mathrm{k}, 74.78 . \mathrm{Na}$
\end{abstract}

One of the most spectacular indications of the Josephson effect is locking of Josephson oscillations to the frequency of external microwaves. As the Josephson frequency is proportional to voltage, the locking leads to appearance of steps at quantized voltages - so called Shapiro steps 1, 2] - in the current-voltage characteristic $(I-V$ curve) of a Josephson junction. At the Shapiro step, the superconducting current flowing through the junction oscillates coherently with the external microwave. In this letter, we describe a strong incoherent junction response to applied microwaves observed in small mesoscopic Josephson tunnel junctions in the presence of thermal fluctuations and dissipative environment.

Small mesoscopic Josephson junctions have received a great deal of interest. They display such peculiar phenomena as the quantum and thermal fluctuation-induced escape 2, 3], Coulomb blockade effects [4], Josephson phase diffusion [5, 6], to name just a few. The significance of these various effects depends on the ratio of the Josephson energy $E_{\mathrm{J}}$ to the charging energy $E_{\mathrm{C}}$ and on the thermal fluctuations. Here, we will deal with properties of moderately small Josephson tunnel junctions, with the ratio of $E_{\mathrm{J}} / E_{\mathrm{C}} \gg 1$, and $E_{\mathrm{J}} \simeq k_{\mathrm{B}} T$, which corresponds to the phase diffusion regime induced by thermal fluctuations.

The Josephson phase diffusion in small junctions has been studied in detail both experimentally [5, 7] and theoretically $[\underline{8},[9]$. Recently, the phase diffusion regime has been observed also in layered high-temperature superconductors [10]. The characteristic feature of this regime is the absence of pure zero-voltage superconducting state accompanied, however, by a hysteresis in the $I-V$ curve. The phase diffusion behavior is a consequence of frequency dependent damping [5], i.e. the dissipation can be rather weak at zero frequency but it reaches a substan-

*Present address: Theoretische Physik III, Ruhr-Universität Bochum, D-44780 Bochum, Germany tial value at frequencies close to the plasma frequency $\omega_{p}$ of the junction. That leads to a diffusion of the junction state with time in the Josephson phase space.

The influence of microwave radiation on Josephson tunnel junctions is, in general, well understood. There are such well-known effects as Shapiro steps mentioned above, photon assisted tunneling of quasiparticles [2], and resonant escape of the Josephson phase [3, 11]. However, the influence of microwave radiation in the Josephson phase diffusion regime got so far very little attention. While being interesting by itself, the microwave-driven phase diffusion is also an implementation of a very general phenomenon as the ac driven diffusion of a particle in a periodic potential [12, 13, 14].

In this Letter, we report on novel behavior induced by microwaves in small Josephson junctions. For low microwave frequencies, the autonomous $I-V$ curves of the studied junctions display a characteristic phase diffusion branch close to zero voltage, which evolves into a pronounced peak shifting to higher voltages as the power $P$ of the microwave radiation increases. We explain this behavior as an enhancement of the Josephson phase diffusion by microwaves. Our analysis carried out for relatively low microwave frequencies $\omega \ll \omega_{p}$ takes into account both incoherent superconducting current and multi-photon absorption (emission). As the frequency of microwave radiation increases, a crossover to well-separated Shapiro steps with a finite slope is observed.

For the experiments, we have fabricated several sub-micron size $\mathrm{Nb} / \mathrm{AlO}_{x} / \mathrm{Nb}$ Josephson tunnel junctions. The junction area was defined in a sputtered $\mathrm{Nb} / \mathrm{AlO}_{x} / \mathrm{Nb}$ trilayer by using electron-beam lithography and reactive ion etching. For insulation of the trilayer edges we used highly cross-linked PMMA [15]. The critical current density of the trilayer is about $220 \mathrm{~A} / \mathrm{cm}^{2}$. We have measured several sub-micron junctions of comparable dimensions, which all showed similar behavior. Measurements presented in this paper have been performed on a junction having the area of 
$0.07 \mu \mathrm{m}^{2}$ as estimated from its normal resistance $R_{\mathrm{T}}$. The Josephson coupling energy calculated from the estimated fluctuation-free critical current of $I_{\mathrm{c}}=150 \mathrm{nA}$ (taken as a half of the current rise at the gap voltage $V=2 \Delta / e$, which is close to the maximum expected Ambegaokar-Baratoff critical current $\left.I_{\mathrm{AB}}=\pi \Delta /\left(2 e R_{\mathrm{T}}\right)\right)$ was $E_{\mathrm{J}}=I_{\mathrm{c}} \Phi_{0} /(2 \pi)=4.9 \cdot 10^{-23} \mathrm{~J}$, where $\Phi_{0}=h /(2 e)$ is the magnetic flux quantum. We estimated the specific capacitance $C_{\mathrm{s}}=46 \mathrm{fF} / \mu \mathrm{m}^{2}$ of the Josephson tunnel barrier by measuring the voltages of Fiske steps in long junctions made of the same trilayer. The charging energy $E_{\mathrm{C}}=e^{2} /(2 C)$ corresponding to the junction capacitance $C$ was about $0.91 \cdot 10^{-23} \mathrm{~J}$, yielding the ratio of $E_{\mathrm{J}} / E_{\mathrm{C}} \approx 5.4$. Measurements have been performed at a temperature $4.2 \mathrm{~K}$, yielding $k_{\mathrm{B}} T=5.8 \cdot 10^{-23} \mathrm{~J}$. Thus, the data presented here approximately fall into the parameter range of $k_{\mathrm{B}} T \approx E_{\mathrm{J}} \gg E_{\mathrm{C}}$.

The measured $I-V$ curves of the junction under microwave irradiation are shown in Fig. 11 We present here data for one polarity of the bias current, as the curves were found to be symmetric with respect to the origin. Microwaves induce a pronounced peak in the $I-V$ curve which shifts towards larger voltages with increasing the microwave power. Simultaneously, we observe the suppression of the superconducting energy gap branch, which we interpret as the well-known photon-assisted quasiparticle tunneling [2]. For low frequency $f$ of microwaves, as shown in Fig. 1(a), both the peak voltage position and its voltage range are much higher than the expected voltage spacing between the Shapiro steps. As the microwave frequency $f$ increases, the low-voltage part of the peak splits into several well-separated steps with finite slope, as seen in Fig. 1(b). The expected Shapiro step voltage spacing here is $\Delta V=\Phi_{0} f \approx 83 \mu \mathrm{V}$, which matches the observed magnitude of the voltage jumps between the low-current features in the $I-V$ curves in Fig. 囵(b).

The voltage of the peak increases with the power $P$ of microwaves. Figure 2 shows the measured voltage $V_{\text {top }}$ at the switching point on the top of the peak as a function of the microwave field amplitude $\propto \sqrt{P}$. One can see that the voltage $V_{\text {top }}$ rises proportionally to the amplitude of the applied microwaves. The current amplitude of the peak decreases with power, as summarized in Fig. 22

Next we turn to the analysis of the $I-V$ curve of a small Josephson junction in the phase diffusion regime. A Josephson junction is characterized by the Josephson phase $\varphi(t)$ satisfying the equation:

$$
\ddot{\varphi}(t)+\alpha \dot{\varphi}(t)+\sin \varphi(t)=j+\eta \sin \omega t+\xi(t) .
$$

In this equation, $j$ is an external dc bias, $\eta$ and $\omega$ are amplitude and frequency of an external microwave radiation, $\xi(t)$ is a random function of time $t$ describing thermal fluctuations. Here, we use dimensionless units, i.e. the time is normalized to $\omega_{p}^{-1}$, the dc bias is normalized to the critical current value $I_{c}$. The dissipative effects
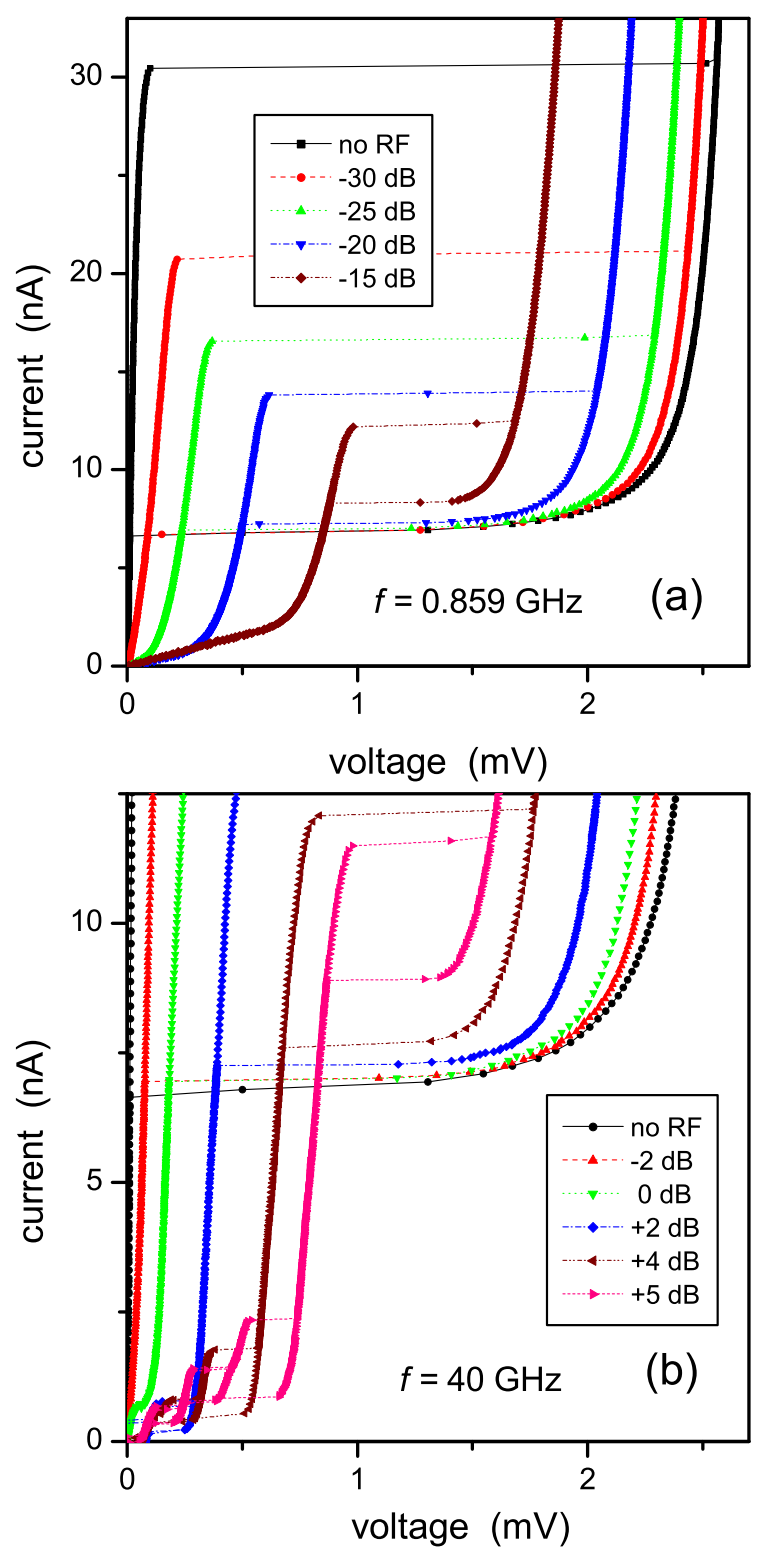

FIG. 1: Current-voltage characteristics of $\mathrm{Nb} / \mathrm{AlO}_{x} / \mathrm{Nb}$ Josephson tunnel junction at $4.2 \mathrm{~K}$. Different curves show the effect of increasing microwave power of the frequency $0.859 \mathrm{GHz}$ (a) and $40 \mathrm{GHz}$ (b). The microwave power levels are referenced to the top of the cryostat.

are described by parameter $\alpha$ that, in general, can be frequency dependent. The presence of hysteretic regime in the observed $I-V$ curves allows to propose a simple model, assuming that the low frequency damping $\alpha_{0}$ determining the quasiparticle current is weak $\left(\alpha_{0} \ll 1\right)$ but the high frequency damping $\alpha$ is large. As the Josephson coupling energy $E_{\mathrm{J}}$ is comparable with the energy of thermal fluctuations $k_{\mathrm{B}} T$, the incoherent superconducting current can be found from the following analysis. In the absence of applied microwave radiation the Josephson 


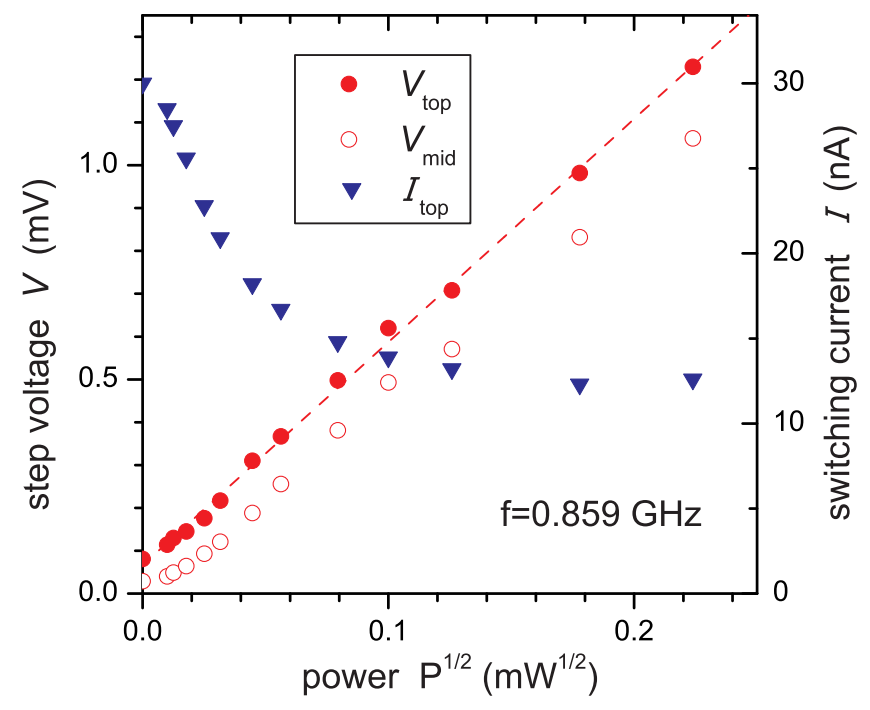

FIG. 2: Dependence of the voltages $V_{\text {top }}$ (solid circles) and $V_{\text {mid }}$ (open circles) and the current $I_{\text {top }}$ (triangles) on microwave field amplitude $\propto \sqrt{P}$. The values of $V_{\text {top }}$ and $I_{\text {top }}$ are measured at the switching point on the top of the microwaveinduced peak on the $I-V$ characteristics. The voltage $V_{\text {mid }}$ refers to the middle (half-height) point of the peak. Dashed line is a linear fit to $V_{\text {top }}(\sqrt{P})$

phase is written as

$$
\varphi(t)=v t+\psi(t)+\varphi_{1}(t)
$$

where $\psi(t)=\frac{1}{\alpha} \int_{0}^{t} \xi(t) d t$ determines the Josephson phase diffusion. Here, the dc voltage drop across the Josephson junction $V$ is normalized to $V_{p}=\Phi_{0} \omega_{p} /(2 \pi)=$ $\frac{1}{2 e} \sqrt{8 E_{\mathrm{J}} E_{\mathrm{C}}}=0.187 \mathrm{mV}$ as $v=V / V_{p}$. In this case, the term $\varphi_{1}(t)$ is expressed through the alternating part of the superconducting current

$$
\varphi_{1}(t)=\frac{1}{\alpha} \int_{0}^{t} d t_{1} \sin \left(v t_{1}+\psi\left(t_{1}\right)\right)
$$

The dc component of the incoherent superconducting current is found as

$$
\begin{gathered}
I_{s}^{0}(v)= \\
\frac{I_{c}}{\alpha} \lim _{T \rightarrow \infty} \int_{0}^{T} \frac{d t}{T} \cos (v t+\psi(t)) \int_{0}^{t} d t_{1} \sin \left(v t_{1}+\psi\left(t_{1}\right)\right)= \\
\frac{I_{c}}{\alpha} \int_{0}^{\infty} d t \rho(t) \sin (v t),
\end{gathered}
$$

where $\rho(t)=\langle\cos (\psi(t)-\psi(0))>$ is the correlation function of a random part of the Josephson phase. This correlation function has been studied in detail for different models in [6, 8] and, in the simplest case of Gaussian random function $\xi(t)$, the $\rho(t)$ has a diffusive form $\rho(t)=\exp (-\delta t)$. Substituting this expression into (4) we obtain $[2,8]$

$$
I_{s}^{0}=\frac{I_{c}}{\alpha} \frac{v}{v^{2}+\delta^{2}}=\frac{I_{c}}{\alpha} \frac{V V_{p}}{V^{2}+\left(\delta V_{p}\right)^{2}} .
$$

This dependence is fitted to our $P=0$ data by using $\delta=0.53$ and $\alpha=4.7$. It is shown in Fig. 3 by dashed line. As expected, it is in a good agreement with the experimentally measured $I-V$ curve in the absence of microwaves. We note that the above value of $\delta$ translates into the high frequency impedance seen by the junction $Z=\delta V_{p} \Phi_{0} /\left(2 \pi k_{\mathrm{B}} T\right) \approx 570 \Omega$, which is not far from the expectation that the bias leads impedance is typically of the order of $100 \Omega$.

In the presence of microwave radiation of a frequency $f=\omega /(2 \pi)$ the Josephson phase can be written as

$$
\varphi(t)=v t+\psi(t)-\frac{\eta}{\alpha} \cos \omega t+\varphi_{1}(t) .
$$

Applying the procedure that is similar to the above treatment of the Josephson phase diffusion without microwave radiation, we obtain the enhancement of superconducting current. This enhancement is due to incoherent absorption (emission) of photons by Josephson phase

$$
I_{s}=\sum_{n=-\infty}^{\infty} J_{n}^{2}\left(\frac{\eta}{\alpha \omega}\right) I_{s}^{0}(v-n \omega)
$$

where $J_{n}(x)$ is the Bessel function of index $n$, and $n$ the number of photons. By making use of the properties of Bessel functions 16] and the limit of multiphoton absorption $(n \gg 1)$ the superconducting current can be written in the following form

$$
I_{s}=\frac{1}{2 \pi} \int_{0}^{\pi} d u\left[I_{s}^{0}\left(v+\eta^{\prime} \cos u\right)+I_{s}^{0}\left(v-\eta^{\prime} \cos u\right)\right],
$$

where $\eta^{\prime}=\eta / \alpha \propto \sqrt{P}$. By substituting expression (5) into (8) we obtain that the superconducting current displays a pseudo-resonant feature, i.e. a peak with downward curvature. The voltage position $V_{0}$ of this peak increases with the power of microwave radiation as $V_{0}=V_{p} \eta^{\prime} \propto \sqrt{P}$. The equation for $I_{s}(V)$ can be presented in the analytical form:

$$
I_{s}=I_{c} \frac{1}{2 \alpha}\left[f_{+}\left(v_{-}\right) f_{+}\left(v_{+}\right)-f_{-}\left(v_{-}\right) f_{-}\left(v_{+}\right)\right],
$$

where we introduced the notations $v_{ \pm}=\left(V \pm V_{0}\right) / V_{p}$ and $f_{ \pm}(x)=\sqrt{\left(\sqrt{x^{2}+\delta^{2}} \pm x\right) /\left(x^{2}+\delta^{2}\right)}$.

Thus, the maximum value of the superconducting current at $V_{0} \gg V_{p} \delta$ weakly decreases with the microwave power $P$ as

$$
I_{s}^{\max }=I_{c} \frac{1}{2 \alpha} \frac{1}{\sqrt{\eta^{\prime} \delta}} \propto P^{-1 / 4} .
$$


The total $I-V$ curve is the sum of the superconducting and quasiparticle currents $I(V)=I_{s}(V)+\alpha_{0} V$ (note here that only the part of the curve with the positive derivative is stable). However, the low frequency damping $\alpha_{0}$ is small and we neglect the quasiparticle current $\alpha_{0} V$ in the following discussion.

Figure 3 presents a comparison of the experimental data with theory for the frequency $f=0.859 \mathrm{GHz}$. Solid lines correspond to the theoretical prediction according to Eq. (9). Our analysis is in excellent agreement with the observations. The theoretical curves in the presence of microwaves are obtained by fitting a single experimental data set for the microwave power level of $P_{0}=-30 \mathrm{~dB}$ and by calculating all other curves according to their power levels relative to $P_{0}$. Note that we used for this procedure the parameters $\delta$ and $\alpha$ obtained from fitting the $P=0$ data to Eq. (5) as mentioned above.

As the microwave frequency $f$ increases, the smaller values of $n$ contribute to the expression (7) and the discrete Shapiro steps having the finite slope appear on the $I$ - $V$ curve, see Fig. 1(b). Our analysis based on Eqs. (8) and (4) can be extended to describe the Josephson phase diffusion in more complex cases. It can be applied to Josephson junctions embedded in a frequency dependent environment, e.g. in the presence of resonances in an external circuit [7], or to a weak Josephson phase diffusion with voltage-dependent parameter $\delta$. As the parameter $\delta$ is small, i. e. $\delta \ll \omega$, the latter regime is of particular interest because the analysis of Eq. (7) shows that at some ac power zero-crossing steps can appear in the $I-V$ curves [17]. Moreover, we have observed such incoherent zero-crossing steps at $4.2 \mathrm{~K}$ for Josephson junctions of an intermediate size with $E_{J} \geq k_{B} T$ [17]. Similar incoherent zero-crossing steps have been also seen in have been also seen in intrinsic junctions based on high-temperature superconductors.

In summary, we have measured the effect of microwaves on mesoscopic Josephson junctions with characteristic energies $E_{\mathrm{C}} \ll E_{\mathrm{J}} \approx k_{\mathrm{B}} T$. In contrast to the behavior of larger Josephson junctions with $E_{\mathrm{J}} \gg k_{\mathrm{B}} T$, which display well-known discrete Shapiro steps due to locking of their Josephson oscillations to microwave frequency, for small junctions we find a smooth and incoherent enhancement of Josephson phase diffusion by microwaves. This enhancement is manifested by a pronounced current peak at the voltage $V_{\text {top }} \propto \sqrt{P}$. We have proposed here a theoretical model for the microwavestimulated phase diffusion which showed excellent agreement with the measured data.

We would like to thank A. Abdumalikov, D. Averin, J. Clarke, E. Ilichev, S. Shitov, and H.B. Wang for useful discussions and acknowledge the partial financial support by the Deutsche Forschungsgemeinschaft (DFG).

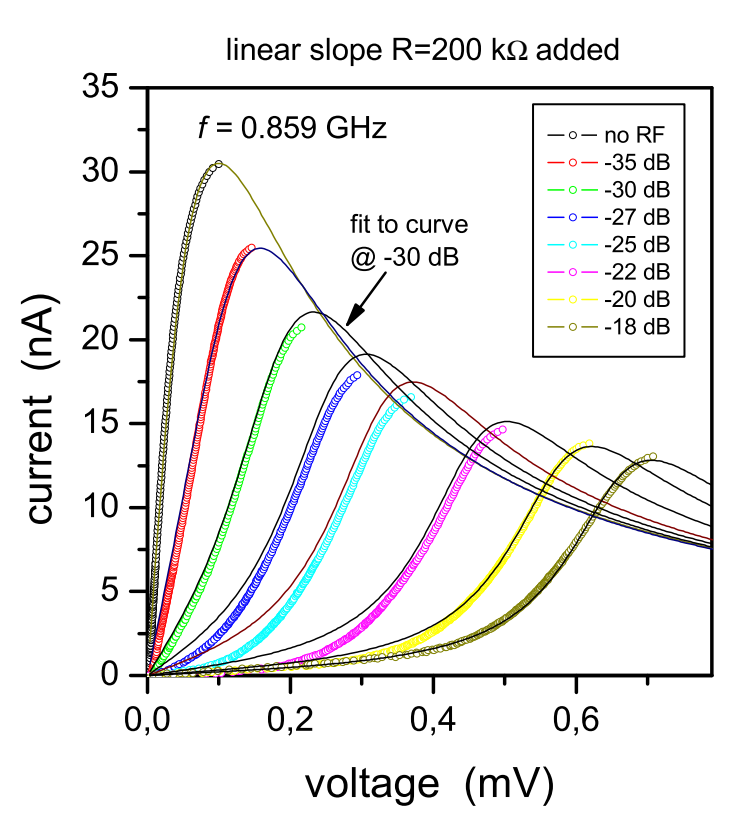

FIG. 3: Comparison between our theoretical model and experiment for the frequency $f=0.859 \mathrm{GHz}$. Dots are experimentally measured data; the dashed line corresponds to Eq. (5) and solid lines correspond to the theoretical prediction according to Eq. (9).

[1] S. Shapiro, Phys. Rev. Lett. 11, 80 (1963).

[2] M. Tinkham, Introduction to Superconductivity, 2nd edition, McGraw-Hill, New York (1996)

[3] M. H. Devoret, J. M. Martinis, and J. Clarke, Phys. Rev. Lett. 551908 (1985); J. M. Martinis, M. H. Devoret, and J. Clarke, Phys. Rev. B 35, 4682 (1987).

[4] D. V. Averin, A. B. Zorin, and K. K. Likharev, Sov. Phys. JETP 61, 407 (1985); D. V. Averin and K. K. Likharev, J. Low Temp. Phys. 62, 345 (1986) .

[5] J. M. Martinis and R. L. Kautz, Phys. Rev. Lett. 63, 1507 (1989); R. L. Kautz and J. M. Martinis, Phys. Rev. B 42, 9903 (1990).

[6] G.-L. Ingold, H. Grabert, and U. Eberhardt, Phys. Rev. B 50, 395 (1994).

[7] D. Vion, M. Götz, P. Joyez, D. Esteve, and M. H. Devoret, Phys. Rev. Lett. 773435 (1996).

[8] G.-L. Ingold and Yu. V. Nazarov, in Single Charge Tunneling, ed. H. Grabert and M. H. Devoret, NATO ASI, Ser. B, Vol. 294 (Plenum, New York, 1991).

[9] H. Grabert, G.-L. Ingold and B. Paul, Europhys. Lett. 44, 360 (1998).

[10] A. Franz, Y. Koval, D. Vasyukov, P. Müller, H. Schneidewind, S. A. Ryndyk, J. Keller, and C. Helm, Phys. Rev. B 69, 014506 (2004).

[11] A. Wallraff, T. Duty, A. Lukashenko, and A. V. Ustinov, Phys. Rev. Lett. 90, 037003 (2003); M. V. Fistul, A. Wallraff, and A. V. Ustinov, Phys. Rev. B 68, 060504(R) (2003). 
[12] R. Bartussek, P. Hänggi, and J. G. Kissner, Europhys. Lett., 28, 459 (1994).

[13] M. V. Fistul, Physical Review E, 65, 046621 (2002).

[14] S. Scheidl and V. M. Vinokur, Physikal Review B, 65, 195305 (2002).

[15] Y. Koval, A. Wallraff, M. Fistul, N. Thyssen, H. Kohlstedt, and A. V. Ustinov, IEEE Trans. Appl. Supercond.
9, 3957 (1999).

[16] M. Abramowitz and I. A. Stegun, (Eds.), Handbook of Mathematical Functions with Formulas, Graphs, and Mathematical Tables, 9th printing. New York: Dover (1972).

[17] Y. Koval, M. V. Fistul and A. V. Ustinov, unpublished. 